\title{
Network-Assisted D2D Discovery Method by using Efficient Power Control Strategy
}

\author{
Hind Albasry and Qasim Zeeshan Ahmed \\ School of Engineering and Digital Arts, University of Kent, Canterbury, CT2 7NT, United Kingdom \\ Email: \{hrja2,q.ahmed\}@kent.ac.uk
}

\begin{abstract}
Neighbour discovery is an important process in device-to-device (D2D) communications. In cellular networks, D2D discovery signals are multiplexed with cellular signals causing in-band emission interference (IEI). IEI degrades D2D user equipments (DUEs) discovery range and cellular user equipments (CUEs) throughput. In this paper, a new discovery method is proposed by applying power control strategy. In this method, DUEs are arranged into two groups depending on whether the received power of a reference signal sent from the based station (BS) to DUEs is larger than a given threshold. A high received reference signal at a DUE indicates strong IEI which may be caused by the DUE to the BS. Then, Group-1 contains DUEs which cause low IEI while Group-2 contains DUEs which cause severe IEI. A new strategy to mitigate IEI is proposed for Group-2. Firstly, CUEs send scheduling information in predefined blocks. Secondly, DUEs estimate the symbols which are orthogonal to CUE. This will assist DUEs to boost their discovery transmission power, reduce IEI and improve the discovery performance.
\end{abstract}

\section{INTRODUCTION}

Cellular mobile communications has been developing rapidly [1]-[12]. Device-to-device (D2D) communication is a promising technology for the next-generation cellular network [13]. It allows devices in proximity to detect each other and communicate directly. In D2D communication, an important process is to discover neighbours [13]. In cellular networks, the discovery resources are multiplexed with uplink physical channel causing in-band emission interference (IEI) [14]. IEI is defined as the interference falling into the non-allocated resource blocks for all component carriers [15]. This interference is caused by the inter-subcarrier transmission due to the propagation and multipath delay of the discovery signals [16]. It is also caused by the cyclic prefix (CP) mismatch between the uplink transmission in the cellular and D2D discovery signal [16]. The IEI further exacerbate when D2D devices are very close to the base station (BS) as compared to cellular user equipment (CUE) [14], [17]. In order to alleviate this problem, power control strategies are proposed in [14]-[18].

Initially, an open-loop power control (OLPC) was introduced to all D2D user equipments (DUEs) in order to control the IEI impact [14]. This strategy led to unnecessary power constraint for some DUEs, which reduced the discovery performance. A new discovery channel structure was proposed in [16], to solve the power control constraints in [14]. This proposed structure added a scheduling information block (SIB) at the centre of the discovery channel. The scheduling information was sent by the CUE to inform DUEs. According to this information, DUEs decided whether to apply OLPC in its own discovery signal transmission or not. However, SIB required a discovery resource block (DRB) for each subframe, making it inefficient in terms of time and frequency resources, e.g. resource units in long term evolution (LTE) systems, as the number of DRBs were limited. In [17], a totally different approach to mitigate IEI was designed. The discovery period was divided into two groups depending on whether the received power of a reference signal $P_{R S R P}$ at a DUE was larger or smaller than a threshold. Generally, DUEs with $P_{R S R P}$ smaller than the threshold was at the cell edge and DUEs with $P_{R S R P}$ larger than the threshold was around the centre of the cell. Therefore, Group-1 was allocated to cell edge DUEs, and Group-2 was allocated to cell centre DUEs. The grouping in this method leads to a reliable cellular channel in Group-1 for CUEs. However, the number of CUEs allowed to use a reliable resources were limited by Group-1. In another study [18], authors identified the symbols where DUE boost the discovery transmission power. This strategy estimates the location of orthogonalityconserved symbols (OCSs). OCSs were defined as the aligned symbols between DRB and physical resource block (PRB) of CUE in a specific sub-frame. These symbols did not have IEI. However, in this method, the DUE only boost the power in OCSs location and used an OLPC in the rest of symbols.

In order to mitigate IEI caused by D2D discovery while not impairing the D2D discovery efficiency in terms of the probability of successful discovery for DUEs, in this paper, a new D2D discovery method is proposed by taking the advantage of dividing DUEs to different groups and adopting SIB to provide information only for necessary groups to use OLPC and OCSs. In this method, DUEs are separated into two groups, based upon whether $P_{R S R P}$ of a DUE is larger than a threshold. Group-1 DUEs do not affect the cellular system, while CUEs are only affected by IEI from DUEs in Group-2. Due to the proximity of DUEs in Group-2 the severe IEI is caused by Group-2 while Group-1 causes negligible IEI. We alleviate the IEI impact in Group-2 to make it more reliable to CUE by utilizing SIB information, and using OCSs estimation. Therefore, we eliminate using OLPC, which degrades the discovery performance. We use OLPC if only the CUE is scheduled to send cellular traffic, and there is no OCSs between the DUEs and CUEs. Our method significantly 


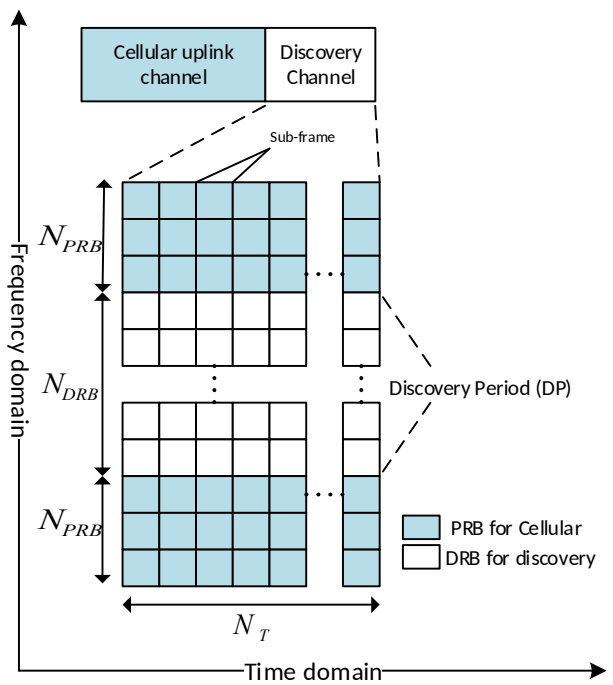

Fig. 1: D2D discovery channel structure

improves D2D discovery performance as compared to conventional techniques. This proposed method does not affect the CUEs received signal at the BS. Furthermore, this method needs less SIB resources than the conventional approach as proposed in [16], which makes it more efficient in terms of discovery resources.

This paper is organized as follows. Section II describes discovery channel resources and IEI impact. Our proposed discovery method is introduced in Section III. Section IV illustrates the performance evaluation and simulation results of our method. Section V finally concludes our study.

\section{DISCOVERY CHANNEL RESOURCES AND IEI IMPACT}

Fig. 1 depicts the D2D discovery channel structure as proposed in 3rd Generation Partnership Project (3GPP) standardization [16]-[18]. As shown in Fig. 1, The discovery channel is composed of $N_{T}$ sub-frames in time domain. DRBs are frequency multiplexed with cellular PRB. $2 N_{P R B} \times N_{T}$ resource blocks are allocated to the CUEs, while $N_{D R B} \times N_{T}$ resource blocks are allocated to the DUEs [16]-[18]. A DUE transmits discovery signal by its allocated DRB and listens to the remaining DRBs to discover neighbours. To be compatible with the existing LTE system, the time duration of a DRB is one time slot of 0.5 milliseconds. Each DRB consists of 12 subcarriers, each having a bandwidth of $15 \mathrm{kHz}$.

Fig. 2 shows the IEI impact of DUE discovery signal to the CUE received signal at the BS. The CUE transmits the information to the BS. D2D discovery exists between a DUE transmitter and a receiver. For a DUE transmitter close to the BS, if the DUE transmit discovery signal with maximum transmission power, severe IEI is caused. One of the proposed solutions to reduce the IEI impact is to control the transmission power of the DUEs.

\section{Proposed BS Aided Device Discovery method}

Fig. 3 describes the discovery channel structure of our proposed method. The discovery period (DP) is divided into

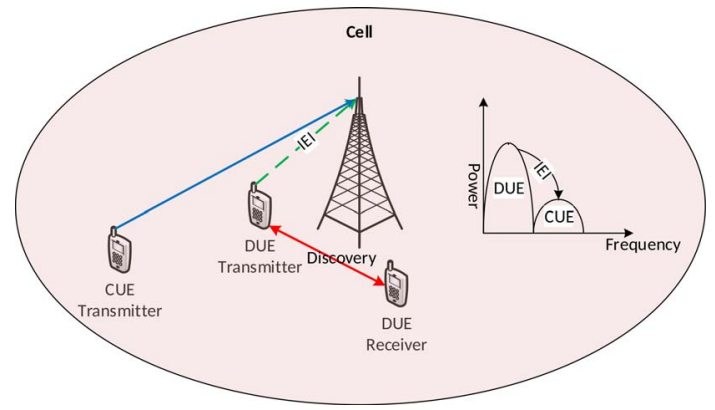

Fig. 2: In-band emission interference in cellular network D2D

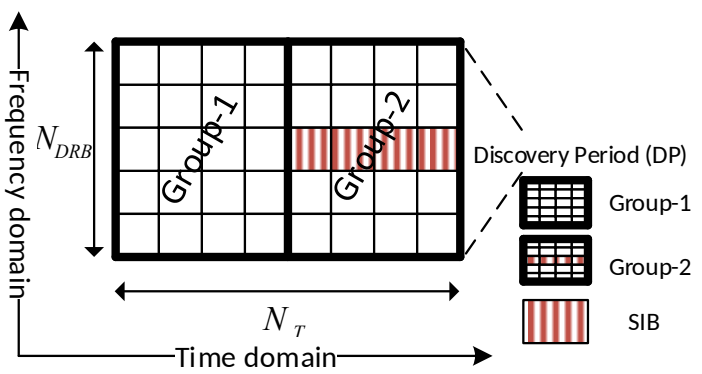

Fig. 3: Discovery proposed model

two time groups, Group-1 and Group-2. In Group-2, a DRB is allocated for each sub-frame in the middle of DP. This DRB is used as SIB. The proposed algorithm is summarized as follows:

Step-1: All DUEs need to be authorized by the network to start the discovery process. DUE sends an authorization request to the network in order to obtain related information such as (Cell-ID, announcing policy, etc.). The BS responds by sending the authorization information to the DUE.

Step-2: DUE calculates the $P_{R S R P}$ as follows

$$
P_{R S R P}=P_{T x}-(P L+\text { Shadowing }),
$$

where $P_{T x}$ is the discovery transmission power of DUE and $P L$ is the path loss between the BS and the DUE. Shadowing is assumed to follow log-normal distribution. The DUE then transmits the discovery request to the BS.

Step-3: After authorization has been done, the BS prepares discovery response messages to the authorized DUEs. These messages contain the discovery authorization code, a valid timer for the discovery, and DRB allocation to the DUE. The resource allocation of DUEs depends on $P_{R S R P}$ information. The DP is divided into two groups in time. Group-1 resources are allocated to DUEs if $P_{R S R P}<$ pre-defined threshold, and Group-2 resources are allocated to DUEs if $P_{R S R P}>$ predefined threshold. This step encloses the DUEs that cause IEI in one group. Group-1 presents the DUEs that are likely to be located far from the BS. Group-2 DUEs are likely to be located close to the BS as shown in Fig. 3.

Step-4: Each DUE starts the discovery procedure by using the DRBs that has been assigned to them by the network (step-3). The DUEs announce the discovery information and 
(monitor or listen to) the rest of the DRBs in order to discover neighbours. The DUEs in Group-1 announce the discovery information without applying any power control strategy as they are far from the BS and the IEI impact is negligible. Meanwhile, the DUEs in Group-2 are subject to a power control strategy in order to reduce the IEI, as they are closer to the BS. Group-2 DUEs start checking the SIB information, which are sent by CUEs. This information contains the scheduling information and CP configuration of the CUEs that will help DUEs to decide their discovery transmission power. DUEs can adjust the transmission power to maximize the neighbourhood discovery, in case there is a scheduled CUE. Furthermore, DUEs estimates the OCS locations by using one of following methods: path loss, round trip delay (RTD) information, timing advance command of BS, and the SIB information. The DUEs boost the discovery transmission power in OCS as these symbols is not affected by IEI. Therefore, Group-2 DUEs only apply OLPC if two conditions are met: There is a scheduled CUE user, and there is no orthogonality between CUE and DUE symbols. Otherwise, the DUE can boost its transmission power to announce its discovery signal no more than predefined maximum power. Accordingly, the discovery transmission power of DUE is calculated as follows: In Group-1 no power control (NPC) is applied. In Group-2, NPC is applied, where there is no scheduled CUE. In this scenario, the power transmitted by the DUE is given by

$$
P_{D U E}^{(k)}=P_{\max },
$$

where $k$ is the DRB index of DUE, and $P_{\max }$ is maximum transmission power.

In Group-2, if CUE is scheduled, the transmission power of DUE for each symbol is adjusted as

$P_{D U E, n}^{(k)}=\left\{\begin{array}{ll}\min \left\{P_{\max }, P_{R x-T h r}+P L^{(k)}\right\}, & \text { if } n \in \mathrm{OCS} \\ P_{\max }, & \text { if } n \notin \mathrm{OCS}\end{array}\right.$,

where $n$ is the DUE symbol index in sub-frame, $P_{R x-T h r}$ is target interference level that the $\mathrm{BS}$ receives, and $P L^{(k)}$ is the path loss between the BS and the DUE in $k$. Finally, the total discovery transmission power for one sub-frame is given by

$$
P_{D U E}^{(k)}=\frac{1}{m} \sum_{n=1}^{m} P_{D U E, n}^{(k)},
$$

where $m$ is the total number of symbols in one sub-frame.

Step-5: After the DUEs discovery procedures, the discovery report then is sent to the BS. The BS terminates the discovery process once the discovery time runs out.

\section{Performance Evaluation and Simulation RESULTS}

In this section, we evaluate the proposed power control strategy in terms of IEI impact on CUEs and the performance of DUE discovery. We compare our proposed method with the conventional strategies as proposed in [16]-[18]. Case-A and
TABLE I: Simulation Parameters

\begin{tabular}{|c|c|}
\hline Parameter & Value \\
\hline Cluster radius & $250 \mathrm{~m}$ \\
\hline UE dropping & Uniform random distribution \\
\hline Noise power density & $-174 \mathrm{dBm}$ \\
\hline Path loss exponent & 4 \\
\hline System Bandwidth & $10 \mathrm{MHz}$ \\
\hline$N_{D R B}$ & 44 \\
\hline Carrier frequency & $2 \mathrm{GHz}$ \\
\hline Shadowing standard deviation & $7 \mathrm{~dB}$ \\
\hline CUE target to BS distance & $50 \mathrm{~m}$ \\
\hline DUE to DUE target & $3 \mathrm{~m}$ \\
\hline RSRP pre-defined threshold [17] & $-67 \mathrm{dBm}$ \\
\hline
\end{tabular}

Case-B in Group-2 in this section denote the performance of our proposed method. Case-A is used if there are scheduled CUEs defined by SIB. In this case, DUE can maximize discovery transmission power only in OCS symbols. Case$\mathrm{B}$ is used if there is no scheduled CUE. In this case, DUE can maximize transmission power to transmit the discovery signal. Case-B will also show us the upper bound which can be achieved when there is no IEI.

\section{A. Evaluation Scenario:}

The simulation parameters are set according to 3GPP standardization. All the parameters are shown in Table-1. DUEs are uniformly distributed in a radius of 250 meters. The maximum transmission power is $23 \mathrm{dBm}$. Small scale fading is modelled by Rayleigh distribution, while log-normal and standard path loss is assumed for large scale fading, respectively [19]. No more than one DRB is allocated to each DUE. In the next sections, we will study the effect of IEI on CUE as well as on DUE.

\section{B. Effect on CUE:}

The impact of DUE on CUE is evaluated by using signalto-interference-plus-noise ratio (SINR) of CUE at the BS. We assume there is a targeted CUE which transmits a cellular signal, and is occupying the PRB located directly next to the DP. The evaluation is based upon worst case scenario. Therefore, the targeted CUE is located at the edge area of each group, separately. Further, $N_{D R B}$ DUEs are transmitting their discovery signal in the same sub-frame as the CUE. $N_{D R B}$ is the maximum number of DUEs allowed to discover neighbours in each sub-frame. The SINR of CUE at the BS is given by

$$
S I N R_{C U E}^{(j)}=\frac{P_{C U E}^{(j)} \cdot\left|h_{C U E, B S}^{(j)}\right|^{2}}{I E I^{(j)}+\sigma^{2}},
$$

where $P_{C U E}^{(j)}$ is the maximum transmission power of targeted CUE, $\left|h_{C U E, B S}^{(j)}\right|^{2}$ is the channel gain between the targeted CUE and BS, $j$ is PRB index of cellular user, and $\sigma^{2}$ is the white Gaussian noise power at the receiver. $I E I^{(j)}$ in $\mathrm{dB}$ for $j t h$ PRB is given by

$$
I E I_{d B}^{(j)}=\sum_{k=1}^{N_{D R B}} P_{D U E}^{(k)}-P_{I E I}^{(k-j)}+H_{D U E, B S}^{(k)},
$$




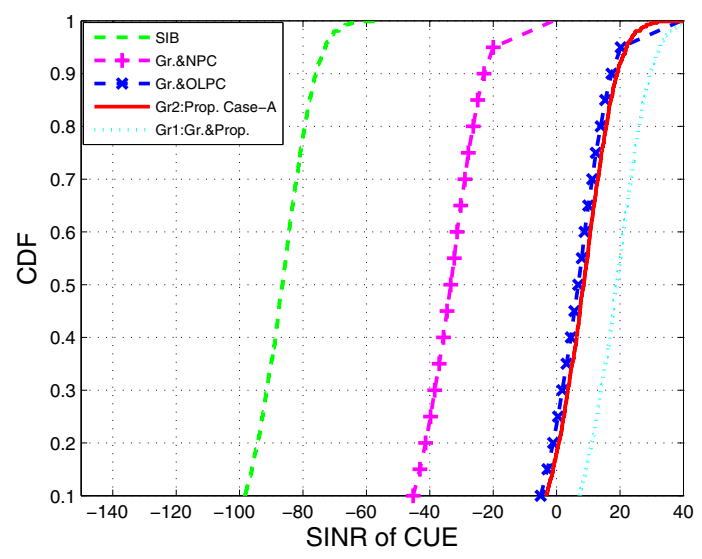

Fig. 4: CDF performance comparisons of received cellular signal $S I N R$ at the BS [Grouping (Gr.), Proposed method (Prop.), Group-1 (Gr1), Group-2 (Gr2)]

where $P_{D U E}^{(k)}$ is the discovery transmission power of DUE as calculated in (2) and (4). $H_{D U E, B S}^{(k)}$ is the channel gain between BS and DUE in $k . P_{I E I}^{(k-j)}$ is the power difference between $j t h$ PRB of the target CUE and $k t h$ DRB of the interfering DUE, given by

$$
P_{I E I}^{(k-j)}=\left\{\begin{array}{lll}
0, & \text { if in } & \text { Group-1 } \\
\bar{P}_{I E I}^{(k-j)}, & \text { if in } \text { Group-2 }
\end{array},\right.
$$

and $\bar{P}_{I E I}^{(k-j)}$ is calculated in [20] as

$$
\bar{P}_{I E I}^{(k-j)}=\left\{\begin{array}{ll}
-21 d B, & \text { if }|k-j|=1 \\
-26 d B, & \text { if }|k-j|=2 \\
-31 d B, & \text { if }|k-j|=3 \\
-36 d B, & \text { if }|k-j|>3
\end{array} .\right.
$$

Fig. 4 shows the cumulative distribution function (CDF) performance of received cellular signal $S I N R$ at BS. In this figure, we compare the IEI impact on targeted CUE under different power control strategies. The required CUE SINR for successfully signal decoding at the BS is $-7.8 \mathrm{~dB}$ [21]. From the figure, it can be observed that only two strategies SIB [16] and Group-2 NPC have decoding level less than $7.8 \mathrm{~dB}$. The performance in these cases are worst as IEI will be severe as no power control technique is applied and DUE operate at maximum power of $23 \mathrm{dBm}$. From the figure, it can be observed that OLPC used in Group-2 and our proposed method in Group-2 have much better performance. In these cases, the CUE signal can be received reliably at the BS. Furthermore, from the figure it can be observed that our proposed method outperforms SIB [16], OLPC [14], and NPC in terms of IEI impact on targeted CUE. Let us now look into the impact of IEI on DUE.

\section{Effect on DUE:}

The impact of CUE on desired DUE is also evaluated by using $S I N R$ as the criterion. We assume there is a targeted D2D pair (a DUE is in listening mode and the other DUE is

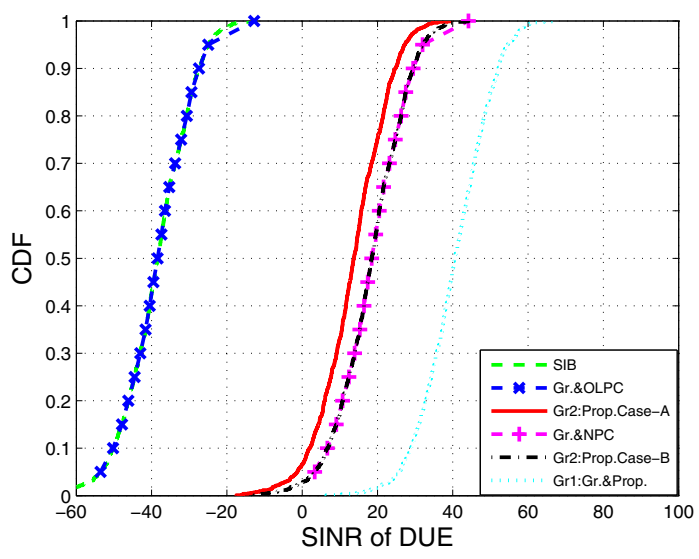

Fig. 5: CDF performance comparisons of received discovery signal SINR at the DUE [Grouping (Gr.), Proposed method (Prop.), Group-1 (Gr1), Group-2 (Gr2)]

in transmitting mode). $\left(N_{D R B}-1\right)$ other DUEs are sending their discovery signal and $2 N_{P R B}$ CUEs are transmitting in the same sub-frame. The worst case scenario, the CUEs are located near the DUE receiver. The DUE SINR is given by

$$
S I N R_{D U E}^{(k)}=\frac{P_{D U E}^{(k)} \cdot\left|h_{D 2 D}^{(k)}\right|^{2}}{I E I^{(k)}+\sigma^{2}},
$$

where $P_{D U E}^{(k)}$ is the discovery transmission power of target DUE in transmitting mode and calculated in (2) and (4). $\left|h_{D 2 D}^{(k)}\right|^{2}$ is the channel gain between target D2D pair. $I E I^{(k)}$ is the interference due to IEI from the CUEs. Fig. 5 depicts CDF of received discovery signal SINR. The DUE is assumed to successfully receive the discovery signal if the $S I N R$ exceeds a given threshold $S I N R_{T h r}$ (assumed $8 \mathrm{~dB}$ in simulation) [21]. From the figure, it can be observed that only SIB [16] and OLPC [14] do not exceed the SIN $R_{T h r}$, which mean that the DUE receiver will not detect successfully the discovery signal. The performance in these cases are worst as OLPC is applied. From the figure, it can be observed that our proposed method in Group-1, Group-2 (case-A, case-B), and NPC have much better performance. In this case the DUE discovery signal can be received reliably at the DUE receiver. It can be observed that our proposed method outperforms SIB and OLPC in terms of the discovery signal detection at the DUE receiver. Even though NPC has same performance with our proposed method case-B, but achieves much better performance in the cellular side as illustrated in previous subsection.

\section{Probability of successful discovery at the DUE receiver:}

The probability of successful discovery is one of metrics to evaluate the discovery performance and is given by

$$
P\left(S I N R_{D U E}^{(k)}>S I N R_{T h r}\right)
$$

Fig. 6 shows the probability of successful discovery at DUE receiver when SIB, OLPC, NPC, and our proposed method 


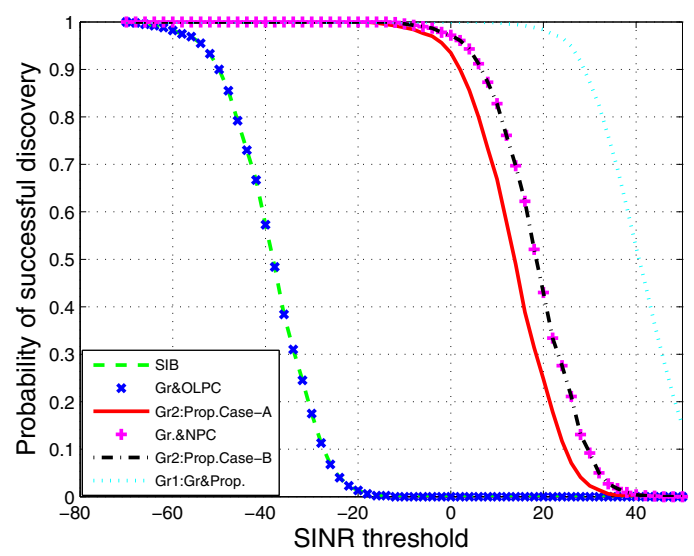

Fig. 6: Probability of successful discovery at the DUE

Group-1 and Group-2 (case-A and case-B) algorithms are employed. The DUE is assumed to have successfully received the discovery signal if the SINR exceeds a given threshold $S I N R_{T h r}$. From the figure, it can be observed the probability of successful discovery under the worst case scenario, is worst for SIB and OLPC which requires a very low SINR threshold to successfully discover the discovery signal. The performance in these cases is the worst as OLPC is applied. From the figure, it can be observed that NPC, our proposed method Group-1 and Group-2 (case-A, case-B) have better performance than SIB and OLPC. Furthermore, the SINR threshold for these methods is higher than SIB and OLPC.

\section{CONCLUSION}

This paper proposed a new D2D discovery method, which mitigated IEI by applying power control strategy. This strategy has the capability to assist DUEs to boost the discovery transmission power, reduce IEI, and improve neighbour discovery performance. Simulation results showed that our proposed algorithm is superior in performance to recently proposed algorithms in this area, such as OLPC, grouping, and SIB. Using simulation results, we verified that $S I N R$ of D2D discovery signal and probability of discovery are improved and SINR of CUE is not affected but slightly improved. The number of discoveries can also be enhanced with our method.

\section{ACKNOWLEDGEMENT}

The first author is supported by funding from the higher committee for education development (HCED) in Iraq. The second author is supported by funding from the European Union's Horizon 2020 project iCIRRUS under grant agreement No 644526.

\section{REFERENCES}

[1] H.Zhu and J.Wang, "Chunk-based resource allocation in OFDMA systems - part I: chunk allocation," IEEE Transactions on Communications, vol. 57, no. 9, pp. 2734-2744, September 2009.
[2] H.Zhu, and J.Wang, "Chunk-Based Resource Allocation in OFDMA Systems - Part II: Joint Chunk, Power and Bit Allocation," IEEE Transactions on Communications, vol. 60, no. 2, pp. 499-509, February 2012.

[3] H.Zhu, "Radio Resource Allocation for OFDMA Systems in High Speed Environments," IEEE Journal on Selected Areas in Communications, vol. 30, no. 4, pp. 748-759, May 2012.

[4] J.Wang, H.Zhu, and N.J.Gomes, "Distributed Antenna Systems for Mobile Communications in High Speed Trains," IEEE Journal on Selected Areas in Communications, vol. 30, no. 4, pp. 675-683, May 2012.

[5] H.Zhu, "Performance Comparison Between Distributed Antenna and Microcellular Systems," IEEE Journal on Selected Areas in Communications, vol. 29, no. 6, pp. 1151-1163, June 2011.

[6] H.Zhu and J.Wang, "Performance Analysis of Chunk-Based Resource Allocation in Multi-Cell OFDMA Systems," IEEE Journal on Selected Areas in Communications, vol. 32, no. 2, pp. 367-375, February 2014.

[7] H.Zhu, and J.Wang, "Radio Resource Allocation in Multiuser Distributed Antenna Systems," IEEE Journal on Selected Areas in Communications, vol. 31, no. 10, pp. 2058-2066, October 2013.

[8] H.Zhu, "On frequency reuse in cooperative distributed antenna systems," IEEE Communications Magazine, vol. 50, no. 4, pp. 85-89, April 2012.

[9] H.Zhu, S.Karachontzitis, and D.Toumpakaris, "Low-complexity resource allocation and its application to distributed antenna systems [Coordinated and Distributed MIMO]," IEEE Wireless Communications, vol. 17, no. 3, pp. 44-50, June 2010.

[10] H.Zhu, B.Xia, and Z.Tan, "Performance Analysis of Alamouti Transmit Diversity with QAM in Imperfect Channel Estimation," IEEE Journal on Selected Areas in Communications, vol. 29, no. 6, pp. 1242-1248, June 2011.

[11] H.Osman, H.Zhu, D.Toumpakaris, and J.Wang, "Achievable Rate Evaluation of In-Building Distributed Antenna Systems," IEEE Transactions on Wireless Communications, vol. 12, no. 7, pp. 3510-3521, July 2013.

[12] T.Alade, H.Zhu, and J.Wang, "Uplink Spectral Efficiency Analysis of In-Building Distributed Antenna Systems," IEEE Transactions on Wireless Communications, vol. 14, no. 7, pp. 4063-4074, July 2015.

[13] K.Choi and Z.Han, "Device-to-device discovery for proximity-based service in LTE-Advanced system," IEEE Journal on Selected Areas in Communications, vol. 33, no. 1, pp. 55-66, Jan. 2015.

[14] D. Li and Y. Liu, "In-band emission in LTE-A D2D: impact and addressing schemes," in IEEE 81st Vehicular Technology Conference (VTC Spring), Glasgow, UK, May 2015, pp. 1-5.

[15] 3GPP TS 36.101, "Evolved Universal Terrestrial Radio Access (EUTRA), User Equipment (UE) radio transmission and reception (Release 13)," July 2015.

[16] W. Hwang, D. Lee, and H. Choi, "A new channel structure and power control strategy for D2D discovery in LTE cellular network," in IEEE Asia-Pacific Conference on Communications (APCC), Pattaya, Thailand, Oct. 2014, pp. 150-155.

[17] D. Kim, Y. Kwak, J. Oh, Y. Kim, and J. Lee, "Discovery resource grouping for D2D discovery for mitigation of in-band emission in LTEAdvanced," in IEEE Globecom Workshops (GC Wkshps), Austin, USA, Dec. 2014, pp. 869-874.

[18] J. Song, D. Lee, W. Hwang, and H. Choi, "A selective transmission power boosting method for D2D discovery in 3GPP LTE cellular system," in IEEE International Conference on Information and Communication Technology Convergence (ICTC), Busan, Korea, Oct. 2014, pp. 267-268.

[19] J. Li, J. Song and Z. Han, "Network connectivity optimization for device-to-device wireless system with femtocells," IEEE Transactions on Vehicular Technology, vol. 62, no. 7, pp. 3098-3109, Sept. 2013.

[20] Y. Kwak, S .Ro, S. Kim, Y. Kim, and J. Lee, "Performance evaluation of D2D discovery with eNB based power control in LTE-advanced," in IEEE 80th Vehicular Technology Conference (VTC Fall), Vancouver, Canada, Sept. 2014, pp. 1-5.

[21] 3GPP TR 36.888, "Study on provision of low-cost Machine-Type Communications (MTC) User Equipments (UEs) based on LTE (Release 12)," June 2013. 